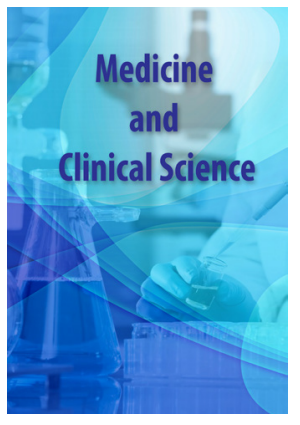

Correspondence

\section{Dr. Manoj V Rao}

Urologic Specialists of Northwest Indiana, 400 West 84th Drive, Merrillville, IN 46410, USA

E-mail:MRao@urologic-specialists.com

- Received Date: 15 Nov 2019;

- Accepted Date: 30 Nov 2019;

- Publication Date: 17 Dec 2019.

Copyright

(C) 2020 Science Excel. This is an openaccess article distributed under the terms of the Creative Commons Attribution 4.0 International license.

\title{
Genetic testing for prostate cancer patients
}

\author{
Manoj V Rao \\ Urologic Specialists of Northwest Indiana, USA
}

\section{Introduction}

Prostate cancer is the one of the most common cancers in men. For years, doctors have relied upon well-studied factors to identify patients who are at higher risk for prostate cancer- older age, African American race, family history, elevated prostate blood test (PSA), or abnormal prostate exam. More recently, there have been evidencebased recommendations from the NCCN (National Comprehensive Cancer Network) for hereditary genetic testing in certain prostate cancer patients. The purpose of this testing is to find men at increased risk for cancers because of mutations in their DNA by doing a blood or saliva test. Mutations are changes in genes, some of which can be associated with cancer. Some mutations you have at birth and are present in every cell in your body while others are acquired after birth and only affect certain parts of the body. Mutations that you have at birth can be hereditary and passed from affected parents to their children.

Men who have prostate cancer with hereditary mutations may have a higher risk for other cancers. Also, some patients with prostate cancer mutations may have more aggressive types of prostate cancer which require closer attention. For years, women with breast cancer have undergone genetic testing to identify mutations in genes associated with increased risks for breast cancer, including the BRCA genes. Many people do not realize that the BRCA genes are also associated with other types of cancer development, including prostate cancer. Men and women can pass a mutation to their children and other blood relatives placing them at risk. There are many gene mutations which can be found in men with prostate cancer. With the idea that genetic testing in prostate cancers is becoming increasingly recommended, our group is interested in helping our eligible patients to undergo this testing and we did this by participating in a research trial for our patients.

\section{Identifying patients at risk}

Our study involved identifying prostate cancer patients who are at risk for genetic mutations, learning how to integrate prostate cancer genetic testing into our office and evaluating doctor and patient experience with this process. The first step was identifying the patient at risk and for whom testing would be indicated. Although there are many ways in which a man with prostate cancer can qualify for genetic testing, the most common qualifying factors are:

1. Metastatic prostate cancer (cancer which has spread)

2. Aggressive type of prostate cancer

3. One or more relatives with metastatic prostate cancer

4. Two or more relatives on the same side of the family with prostate cancer or breast cancer

5. Ashkenazi Jewish Ancestry

6. Family history of certain rare cancers (such as ovarian or pancreatic cancer)

Once we identified patients who are eligible, we would have a discussion to see if they are interested in undergoing genetic testing and provide more information.

\section{Considerations with genetic testing}

We discussed with patients the variety of issues with genetic testing because of its far-reaching implications. It is remarkable that this type of testing can help identify patients and their family members who may be at risk for cancers which they do not currently have. With closer monitoring and lifestyle changes, perhaps we can help catch cancers earlier and also address ways in which they could possibly reduce the chances of developing cancer. Some patients can develop anxiety awaiting results for these tests and their family members may also not want them to undergo this testing due to fear of having a mutation. Some people may be concerned that genetic information may be used against them, but the Genetic Information and Nondiscrimination Act (GINA) of 2008 protects us from genetic discrimination in health insurance and employment. The legislation does not protect patients seeking long term care, disability or life insurance. It is uncertain if genetic information or the presence of prostate cancer alone would be the main problem in obtaining such coverage.

When testing is performed, results can be positive for a mutation that can increase risk for cancer, negative with no mutation or problem identified, or a variant of uncertain significance. Although a variant of uncertain significance is a change in the genetic code, there is not enough evidence to decide whether that change can cause harm or not. With time, only 
about one in ten $(10 \%)$ of these uncertain variants will be considered problematic. For this reason, a person with a variant of uncertain significance will need to stay in touch with their doctor to find out if there is a definitive answer.

While results of our study are still pending, we expect up to $15 \%$ of our patients who undergo genetic testing will test positive for a mutation based on prior studies. In our patients who do have DNA mutations associated with cancer, we are fortunate to have a medical geneticist in our region, Dr. Janice Zunich, and genetic counselors available to help guide families to understanding their options for further testing and what to do next. For the patients with genetic mutations, drugs are being developed to target mutations and this may give them a better way to fight prostate cancer.

\section{The Future of Prostate Cancer}

Recently, a newer class of drugs has been found to improve cancer response rates in men who have specific gene mutations in comparison to currently available treatments. The FDA has designated some of these newer targeted drugs as a breakthrough therapy as they have demonstrated substantially better results in research studies than what we currently offer. Many cancers - ovarian, breast, colon and lung- have a variety of targeted treatments and with the identification of DNA mutations in patients with prostate cancer, we also hope to aid the search for newer medications for our patients. The era of personalized cancer treatment is upon us and the best way to find who is eligible for these cutting-edge treatments is to incorporate genetic testing into patient care. 